\title{
Comparison of experimental tests and theory for a rectangular two-channel dielectric wakefield accelerator structure
}

\author{
S. V. Shchelkunov, ${ }^{1}$ T. C. Marshall, ${ }^{2}$ G. Sotnikov, ${ }^{2,3}$ J. L. Hirshfield, ${ }^{1,2}$ Wei Gai, ${ }^{4}$ M. Conde, ${ }^{4}$ J. Power, ${ }^{4}$ \\ D. Mihalcea, ${ }^{4}$ and Z. Yusof ${ }^{4}$ \\ ${ }^{1}$ Yale University, New Haven, Connecticut 06511, USA \\ ${ }^{2}$ Omega-P, Inc., New Haven, Connecticut 06510, USA \\ ${ }^{3}$ NSC Kharkov Institute of Physics and Technology, 61108 Kharkov, Ukraine \\ ${ }^{4}$ Argonne National Laboratory, Argonne, Illinois 60439, USA \\ (Received 22 November 2011; published 27 March 2012)
}

\begin{abstract}
Experimental results are reported for test beam acceleration and deflection in a two-channel, cm-scale, rectangular dielectric-lined wakefield accelerator structure energized by a $14-\mathrm{MeV}$ drive beam. The dominant waveguide mode of the structure is at $\sim 30 \mathrm{GHz}$, and the structure is configured to exhibit a high transformer ratio $(\sim 12: 1)$. Accelerated bunches in the narrow secondary channel of the structure are continuously energized via Cherenkov radiation that is emitted by a drive bunch moving in the wider primary channel. Observed energy gains and losses, transverse deflections, and changes in the test bunch charge distribution compare favorably with predictions of theory.
\end{abstract}

DOI: 10.1103/PhysRevSTAB.15.031301

PACS numbers: 41.75.Lx, 41.60.Bq

\section{INTRODUCTION}

There is steadily growing interest within the accelerator community towards exploration of dielectric-loaded single- or multiple-channel structures that can support wakefields driven by either a single electron bunch or a periodic train of bunches. This interest provided strong incentive for research which we report in this paper. A variety of dielectric configurations are being explored by different groups [1-27]. Expectations are that larger magnitudes of surface fields, as compared with metal structures, can be tolerated by dielectric-lined structures $[2,3]$. Wakefields can be spatially localized and move at near-light velocity in a vacuum channel surrounded by dielectric. These features ensure that exposed dielectric surfaces are subjected to high field magnitudes for only brief intervals of time, thereby reducing the likelihood for breakdown and permanent structure damage.

Our group and collaborators have investigated a rectangular two-channel dielectric-lined accelerator module that supports wakefields having frequencies of a few tens of GHz. The motivation behind using two-channel structures comes from a simple observation that a single-channel dielectric-lined module has a limitation [4-7] for achieving high transformer ratio (TR), unless provisions are made for bunch trains whose individual bunches have charges conforming to prescribed rules [8-11]. Moreover, if a singlechannel structure is driven by a single drive bunch, a carefully profiled bunch distribution is needed within this

Published by the American Physical Society under the terms of the Creative Commons Attribution 3.0 License. Further distribution of this work must maintain attribution to the author(s) and the published article's title, journal citation, and DOI. bunch to achieve a TR above 2:1. On the other hand, twochannel modules can embody a high TR without imposing any complex set of requirements on the drive bunch or drive train. In two-channel structures, a spatial distribution of wakefields can be sustained that causes the acceleration gradient in one of the channels (henceforth referred to as the test or acceleration channel) to be much higher than the deceleration gradient in the other channel (normally referred to as the drive channel). However, achievement of high TR is subject to limitations dictated by bunch stability requirements for both the accelerated and drive bunches, or drive trains [1,12-14]. Recently, we explored a version of the two-channel structure with favorable symmetry, namely, a coaxial dielectric wakefield accelerator $[12,15]$; in this structure, lateral forces on the test bunch vanish to lowest order.

In this paper we provide a comparison of experimental results with predictions of a theoretical model. To the best of our knowledge, this is the first experiment to test wakefields in a single composite structure comprising two dielectric-lined coupled channels. The goal of the experiment was to excite the structure with a single drive bunch that moves within and is well aligned with the drive channel (Fig. 1), and to probe the wakefield set up by its passage in the test channel using a test bunch, also with well-known parameters. The delay between bunches was adjusted to probe the fields at different distances behind the drive bunch. As theory predicts, the dynamics of the test bunch is dictated mostly by longitudinal and transverse forces $F_{z}$ and $F_{x}$ that arise because of the corresponding electric and magnetic components of the wakefield. While the longitudinal force changes the test bunch energy, the transverse force deflects the bunch horizontally. Both effects are well observed and quantified on the spectrometer 


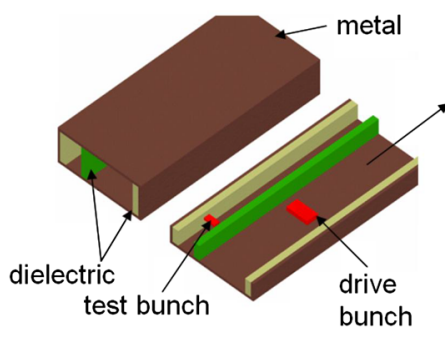

(a)

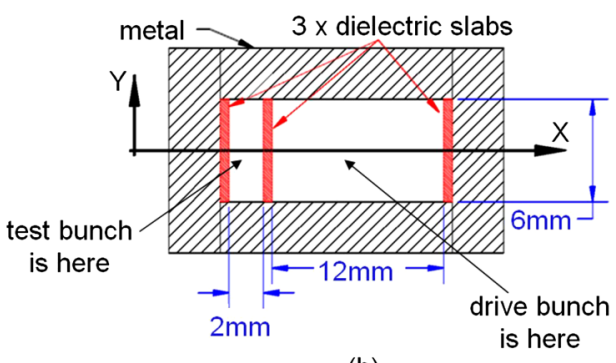

(b)

FIG. 1. (a) Module schematic, and (b) cross section of the apparatus with the channel dimensions. The slab thicknesses (from left to right] are 1.25, 2.3, and $1.06 \mathrm{~mm}$ (manufactured by Euclid Techlabs LLC).

screen, thereby allowing one to find experimentally the strength of the wakefields in a straightforward manner and later make comparisons with the model predictions. Thereby, we shall provide evidence that the simulation and analytical tools and models are adequate, and our understanding of the interaction between the wakefields and bunches is valid.

The structure cross section is shown in Fig. 1. Both dielectric-lined channels are $10 \mathrm{~cm}$ long; the entire apparatus is longer because of a stainless steel mask in front to collimate the bunches. The dielectric is cordierite with a dielectric constant of 4.76 .

The predicted TR is in slight excess of 12:1. The maximum acceleration is predicted to be $6 \mathrm{MV} / \mathrm{m}$ for a $50 \mathrm{nC}$ drive bunch at a distance $\sim 8.5 \mathrm{~mm}$ behind the drive bunch. The drive bunch excites several hybrid modes, mainly pumping energy into $\mathrm{LM}_{31}, \mathrm{LE}_{11}, \mathrm{LM}_{21}$, and $\mathrm{LM}_{11}$ modes, presented here in a descending order based on the amount of power they receive from the drive beam. The $\mathrm{LM}_{31}$-mode eigenfrequency is $\sim 30 \mathrm{GHz}$.

The module structure was tested at Argonne Wakefield Accelerator facility (AWA). The experimental layout is shown in Fig. 2.

Both the drive and the test bunch are produced at the same rf photocathode and accelerated to $\sim 14 \mathrm{MeV}$ in the same rf linear accelerator. The drive bunch is produced on the axis of the cathode; its trajectory almost coincides with the beam-line axis with which the apparatus drive channel is aligned (that is, the middle of the drive channel is put on the beam-line axis). The test bunch is produced off axis so that it may enter the test channel which is located off axis of the beam line. The position and focusing of the test bunch are determined by the same controls that focus and guide the drive bunch [16].

Figure 3 presents some photos of the dielectric wakefield accelerator (DWFA) apparatus.

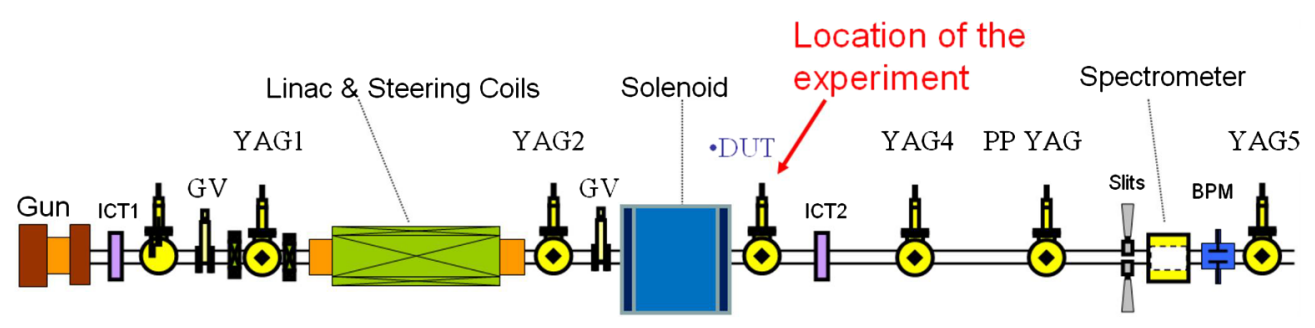

FIG. 2. Experimental layout.

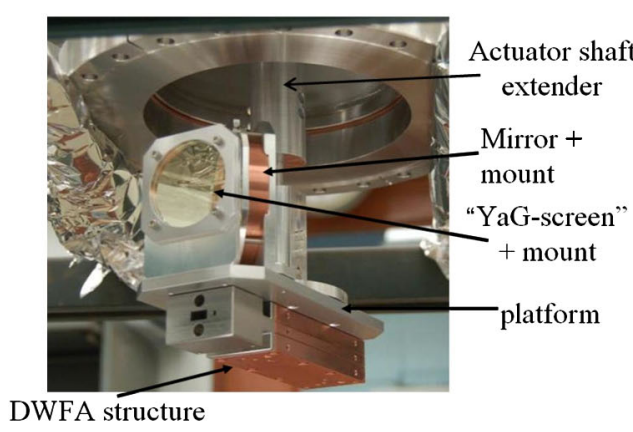

(a)

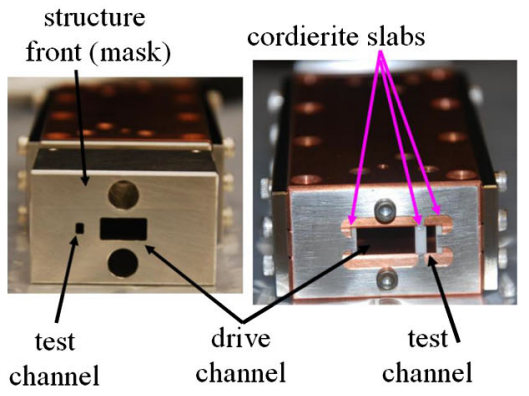

(b)

(c)

FIG. 3. (a) DWFA apparatus attached to an actuator before it is placed in the vacuum chamber; (b) front view (the apparatus is preceded by a mask to protect the dielectric slabs from the beam halo); and (c) back view of the accelerator structure. 
It is recognized that two-channel rectangular structures of small height are prone to bunch instability and will cause a bunch deflection due to intrinsic asymmetry. Our analysis has shown that the structure length should not exceed $10 \mathrm{~cm}$ for the test bunch to clear the test channel without hitting the walls. The maximum deflection is caused in the horizontal plane by a relatively large $F_{x}$ force of $\sim 0.8 \mathrm{MV} / \mathrm{m}$ (if the $50 \mathrm{nC}$ drive is used), but the $F_{y}$ force is small and does not contribute much to test bunch deflection. We emphasize that these limitations do not prevent us from studying the basic physics. Also, we point out that the device may find application as a fast "kicker" [17].

\section{NUMERICAL SIMULATIONS}

Table I shows the design parameters of the two-channel dielectric-lined wakefield accelerator module, as settled upon through numerical studies $[16,18]$ using the analytical theory $[28,29]$ and the CST STUDIO code. For the numerical calculations that used the analytical theory we developed codes compiled by Intel Visual Fortran (IVF). The results obtained using the CST STUDIO software compare favorably with those obtained using the IVF codes at relatively short distance (of order several wavelengths of the operating mode).

Table II shows the amount of power going into the eigenmodes if the module is excited by a $50 \mathrm{nC}$ drive bunch. As already stated, $\mathrm{LM}_{31}, \mathrm{LE}_{11}, \mathrm{LM}_{21}$, and $\mathrm{LM}_{11}$ are the modes into which the bunch radiates most of its power. Figure $4 \mathrm{a}$ presents the transverse $E_{z}$ amplitude profile of some of the modes.

The wakefield is a superposition of LSM and LSE mode wave functions; the composite accelerating force $F_{z}$ is shown in Fig. 4(b); Fig. 5(a) presents the map of the composite axial force $F_{z}$ as a function of $x$ and $z$ in the plane $y=0$.

Depending on the relative delay between the drive and test bunches, the length of the test bunch, and the drive bunch charge, the test bunch may either acquire an additional energy spread, or have its energy spread reduced. In particular, a monoenergetic test bunch with rms length $\sim 2 \mathrm{~mm}$ will experience about 6\% energy spread after traveling $10 \mathrm{~cm}$, obtained from the analysis of different trajectories [see Fig. 5(b)]. In the calculations presented in Fig. 5(b), the initial axial position of test particles is at the first maximum of the accelerating field $z=8.454 \mathrm{~mm}$ [see Fig. 4(b)]; trajectories are shown of 9 particles with their horizontal coordinates being either $x_{1}, x_{2}$, or $x_{3}$, and vertical coordinates being either $y_{1}, y_{2}$, or $y_{3}$, where $x_{1}=$ $x_{a c}-x_{a} / 2, \quad x_{2}=x_{a c}, \quad x_{3}=x_{a c}+x_{a} / 2, \quad y_{1}=-y_{a} / 2$, $y_{2}=0$, and $y_{3}=y_{a} / 2$; and $x_{a c}$ is the $x$ coordinate of the accelerating channel center $\left(x_{a}=1 \mathrm{~mm}, y_{a}=1 \mathrm{~mm}\right)$. More details will be given later, when we present analysis of the experimental data and comparison with the model predictions.

The deflecting forces in the horizontal $(x)$ direction can be large [see Fig. 6(a)]. However, they still permit test

TABLE I. Parameters of the two-channel dielectric-lined wakefield accelerator module.

\begin{tabular}{lc}
\hline \hline $\mathrm{LSM}_{31}$ design mode eigenfrequency (for $v_{\text {phase }}=c$ ) & $30 \mathrm{GHz}$ \\
Drive channel dimensions & $12 \times 6 \mathrm{~mm}$ \\
Accelerating channel dimensions & $2 \times 6 \mathrm{~mm}$ \\
Transformer ratio & $12.6: 1$ \\
Dielectric [cordierite] slab \#1 thickness & $1.24-1.25 \mathrm{~mm}$ \\
Dielectric [cordierite] slab \#2 thickness & $2.29-2.3 \mathrm{~mm}$ \\
Dielectric [cordierite] slab \#3 thickness & $1.05-1.06 \mathrm{~mm}$ \\
Dielectric relative constant & 4.76 \\
Drive bunch size, $\sigma_{x} \times \sigma_{y} \times \sigma_{z}$ & $3 \times 1 \times 2 \mathrm{~mm}$ \\
Bunch energy entering apparatus & $\sim 14 \mathrm{MeV}$ \\
Drive bunch charge & $10-50 \mathrm{nC}$ \\
Number of drive bunches & 1 \\
\hline \hline
\end{tabular}

TABLE II. Eigenfrequencies of two-channel structure and radiation power for the Gaussian distribution of charge within the $50 \mathrm{nC}$ drive bunch.

\begin{tabular}{lcclcc}
\hline \hline Mode & Frequency, GHz & Power, MW & Mode & Frequency, GHz & Power, MW \\
\hline LSM $_{11}$ & 24.79 & 0.52 & LSE $_{11}$ & 19.96 & 2.5 \\
LSM $_{21}$ & 29.97 & 1.07 & $\mathrm{LSE}_{21}$ & 39.07 & 0.135 \\
$\mathrm{LSM}_{31}$ & 30.00 & 2.41 & $\mathrm{LSE}_{31}$ & 44.68 & $9.6 \times 10^{-2}$ \\
$\mathrm{LSM}_{41}$ & 53.61 & $4.36 \times 10^{-2}$ & $\mathrm{LSE}_{41}$ & 45.40 & 0.26 \\
$\mathrm{LSM}_{51}$ & 75.42 & $8.36 \times 10^{-5}$ & $\mathrm{LSE}_{51}$ & 72.99 & $1.94 \times 10^{-4}$ \\
\hline \hline
\end{tabular}




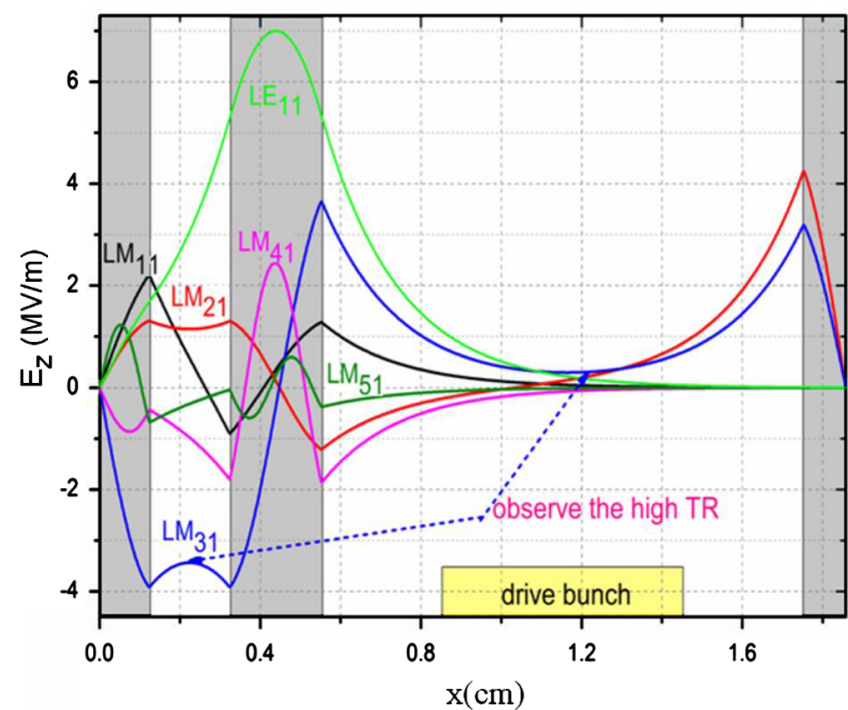

(a)

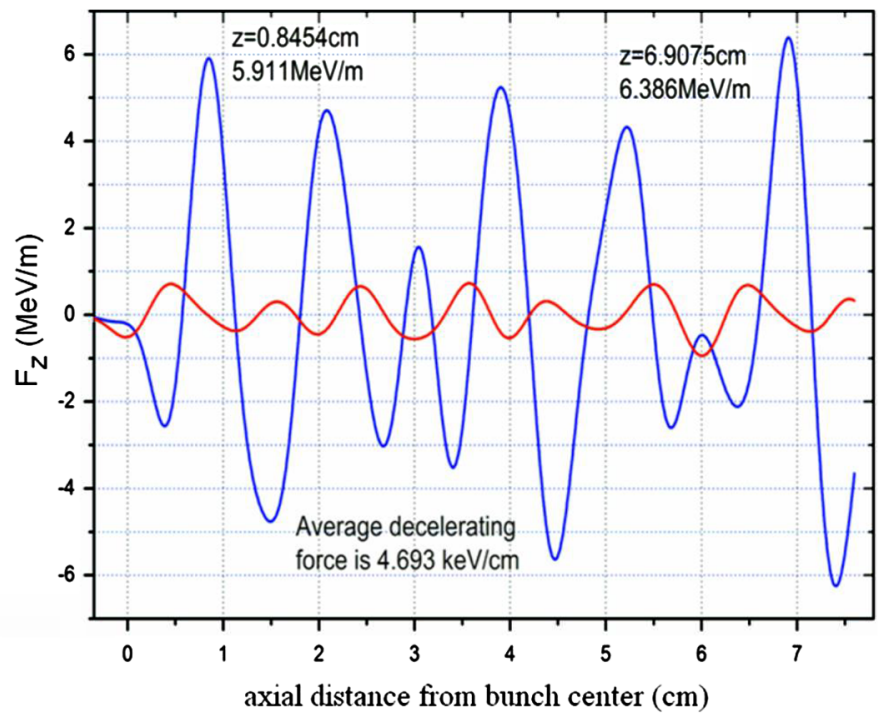

(b)

FIG. 4. (a) Some of the modes which may be excited by a drive bunch. The resulting wakefield is a composite $F_{z}$ force on the test bunch as shown in (b). These curves are given for a $50 \mathrm{nC}$ drive bunch. The curve with the large magnitude in the narrow channel is the accelerating force acting on the test bunch, and the curve with the smaller magnitude in the wide channel is the decelerating force acting on the drive bunch.

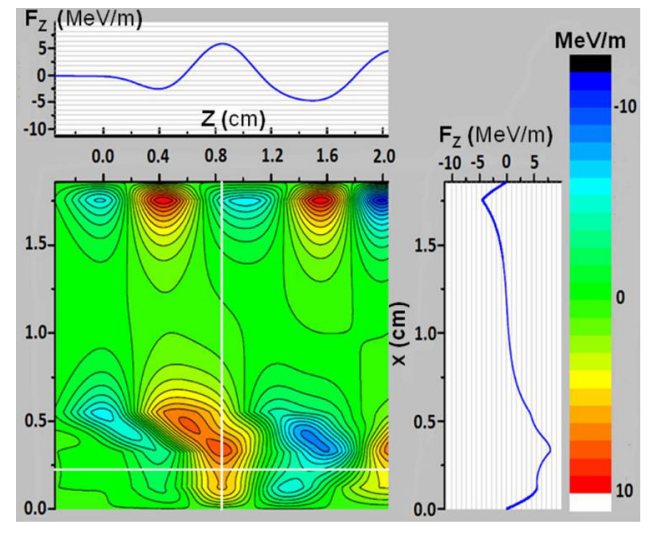

(a)

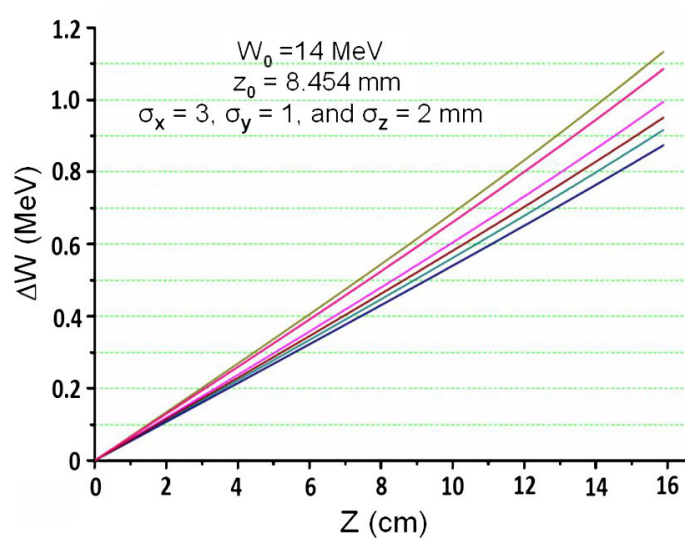

(b)

FIG. 5. (a) The composite axial force $F_{z}(z, x)$ in the plane $y=0$. The middle of the test bunch is at the white cross-hair (IVF computations at Kharkov Institute). (b) Trajectories of test particles for a monoenergetic test bunch with the initial rms length $\sim 2 \mathrm{~mm}$ (see comments in the text).

bunch transmission without interception along the $10 \mathrm{~cm}$ long module as demonstrated by Fig. 6(b). There the horizontal positions of test particles are shown as they move along the structure. The initial energy is taken to be $14 \mathrm{MeV}$. A few test particles are tracked in the wakefield set up by a $50 \mathrm{nC}$ drive bunch, each test particle having its initial axial position $z=0.8454 \mathrm{~cm}$, which corresponds (see Fig. 4) to the maximum of accelerating force $F_{z}$.

The net deflecting forces in the vertical $(y)$ direction are nearly absent in the vicinity of the center of test channel; the nature of vertical forces, $F_{y}$, is either focusing or defocusing depending on the test bunch location. However, the influence of the vertical forces on the bunch dynamics is substantially less than the horizontal forces as demonstrated by Fig. 7. There the vertical positions of test particles are shown as they move along the structure. The initial energy is taken to be $14 \mathrm{MeV}$. A few test particles are tracked in the wakefield set up by a $50 \mathrm{nC}$ drive bunch, each test particle having its initial axial position $z=$ $0.8454 \mathrm{~cm}$, which corresponds (see Fig. 4) to the maximum of the accelerating force $F_{z}$. 


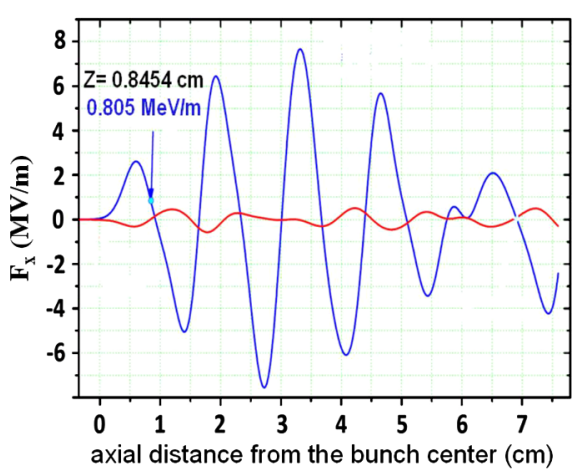

(a)

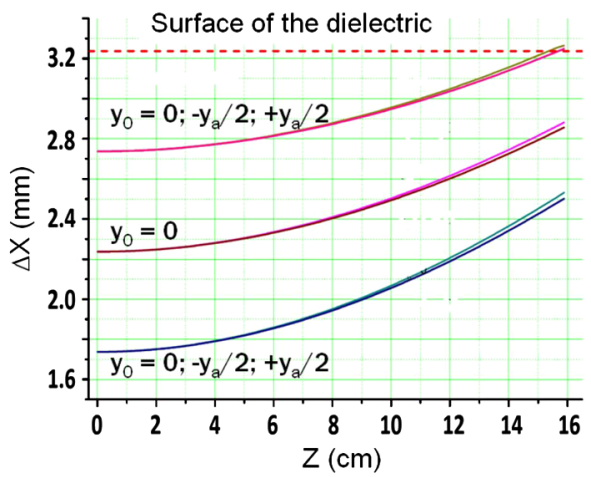

(b)

FIG. 6. (a) Axial profile of the composite horizontal force $F_{x}(y=0)$ along the center of the acceleration channel (blue curve) and the center of the drive channel (red); and (b) the horizontal $(x)$ position of test particles as they move along the structure.

Given the results listed above, the length of module for this proof-of-principle experiment was chosen to be $10 \mathrm{~cm}$.

\section{DESIGN DETAILS AND BUNCH TRANSPORT ISSUES}

Critical aspects of the apparatus included means for precise assembly of the rectangular two-channel DWFA module. Figure 8 shows how all three cordierite slabs are

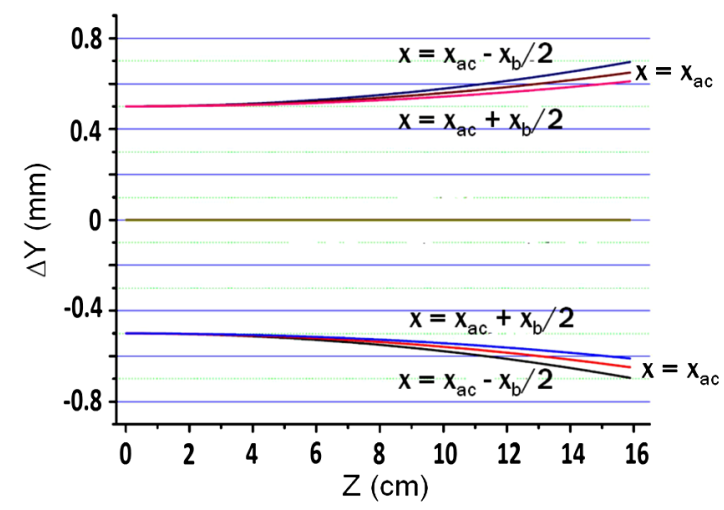

FIG. 7. The vertical ( $y)$ position of test particles as they move along the structure. positioned with high accuracy in a copper block to form the structure, wherein shallow protrusions capture the slabs. In order to position the dielectric slabs with high accuracy, the following precautions were taken: (a) at a few locations along its length the structure has triplets of holes the depth of which ensures that a thin wall is formed between the bottom of each hole and the inner volume of the apparatus; (b) a steel form is inserted, whose protrusions reproduce precisely the slabs dimensions, and are located as far as the slabs should be. The pushing screws (top) are used to produce another set of protrusions in the copper blocks, each facing inward, as shown in (d), so as to capture the dielectric slabs when inserted as shown in (c).

A time-delayed test bunch is produced off axis on the same photocathode where the drive bunch was produced (Fig. 9). The test bunch is made by a second laser pulse that is formed by diversion off a splitter from the main pulse used to create the drive bunch. The test bunch propagates off axis, and its position and focusing is determined by the same controls (linac, magnets) that guide and focus the drive bunch. The yttrium aluminum garnet (YAG) screen after the last bending solenoid serves as a readout for the spectrometer; the vertical size is (by the virtue of design at AWA) totally dominated by the energy spread. Simulations indicated that a test bunch can be delivered to the required location while the drive bunch is still

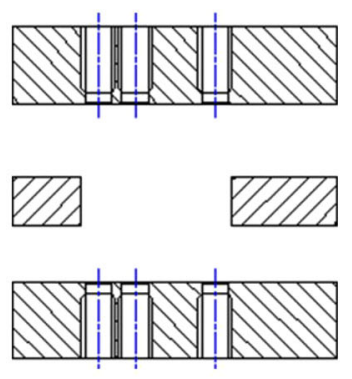

(a)

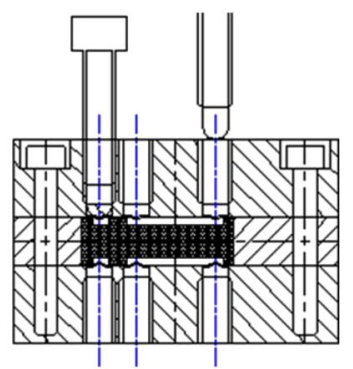

(b)

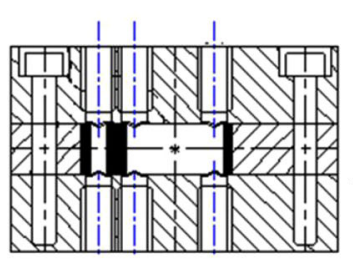

(c)

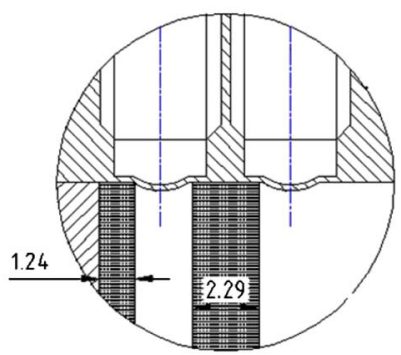

(d)

FIG. 8. Sequence of steps to ensure accurate positioning of slabs (see description in the text). 


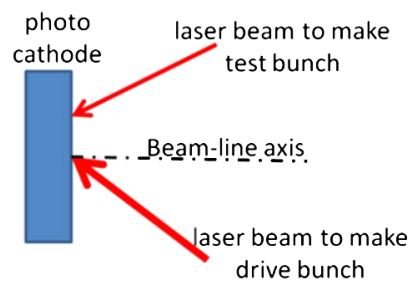

(a)

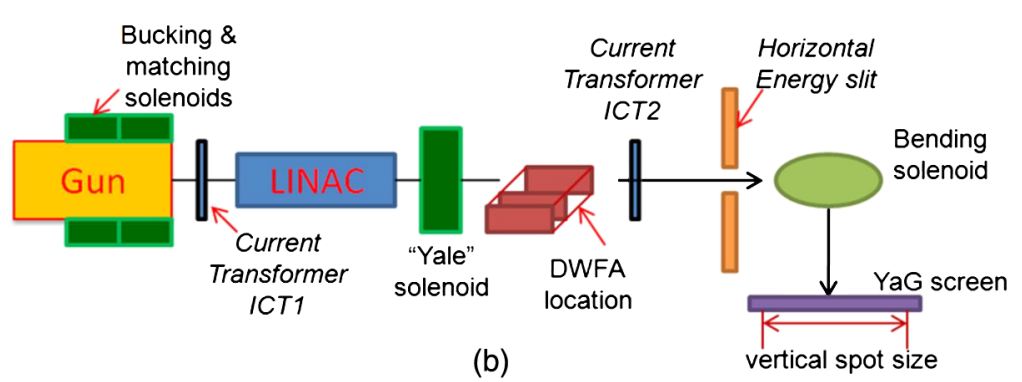

(b)

FIG. 9. (a) Time-delayed test bunch is produced off axis on the same photocathode where the drive bunch was produced. (b) Beamline elements essential to guide and focus both bunches; steering coils and beam position monitors (YAG screens) are not shown.

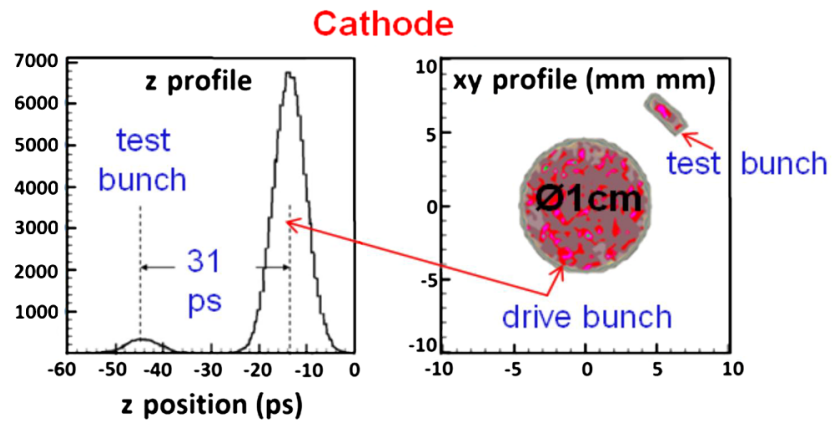

(a)

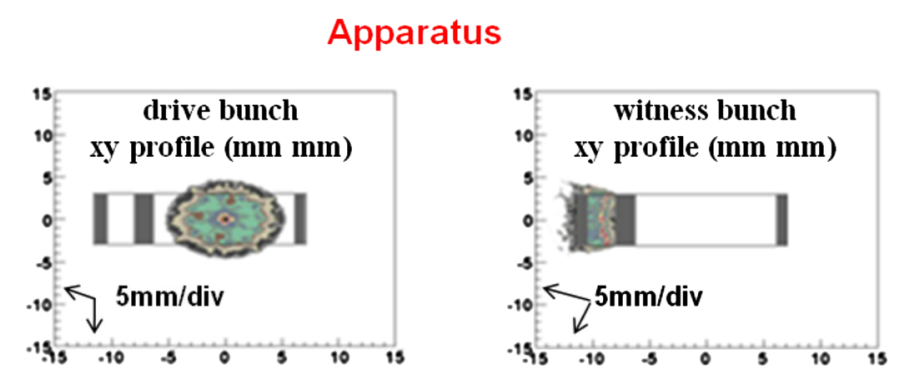

(b)

FIG. 10. Simulations indicate that both bunches produced at the cathode (a) can be guided and focused to the correct locations when delivered to the apparatus (b). The simulation is done for $10^{5}$ particles [the left graph in (a) shows the number of particles vs initial $z$ coordinate for both bunches].

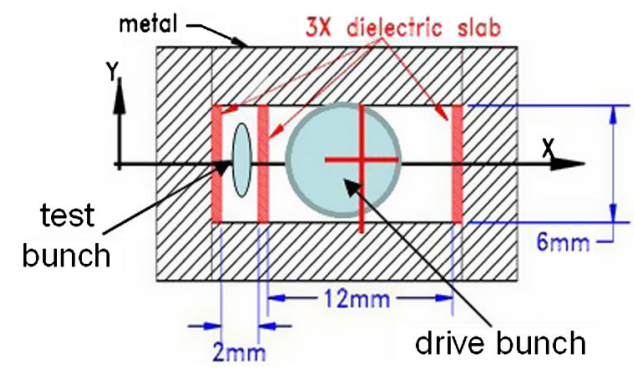

FIG. 11. In the measurements described in this article, the drive bunch was typically located away from the drive channel center axis by $2-3 \mathrm{~mm}$ (see explanations in the text).

focused and positioned as desired (Fig. 10); however, each time the gun phase or delay between the bunches is adjusted, a careful optimization is required to have both bunches transmitted through the apparatus.

Given the narrow size of the test channel preceded by a mask to collimate the beams, the transmission of the test bunch requires both accurate positioning and angular alignment of the structure. Under these circumstances, it was found that the test bunch can be transmitted only when the solenoid does not deliver too strong focusing. This, in turn, did not allow us to use drive bunches with charges exceeding $15 \mathrm{nC}$; otherwise the underfocused drive bunch would partially leak into the test channel and prevent imaging of the test bunch on the spectrometer screen. Another complicating factor is that the horizontal separation between bunches was measured to be typically $7 \mathrm{~mm}$. Given that the spacing between the centers of the two channels is $9.3 \mathrm{~mm}$, the drive bunch was typically located away from the drive channel center axis by $2-3 \mathrm{~mm}$ (see the schematic in Fig. 11.) These practical difficulties indicate that two-bunch schemes are better tested if a facility is equipped with two guns, and two separate beam lines to produce and manipulate the beams independently.

\section{EXPERIMENTAL RESULTS VS MODEL PREDICTIONS: CHANGES IN BUNCH ENERGY AFTER ACCELERATION}

Data were collected for three different delays between the drive bunch and the test bunch, namely $\sim 5.7, \sim 10.7$, and $\sim 21.7 \mathrm{~mm}$. The typical drive bunch charge at the listed delays varied from shot to shot from 8 to $10 \mathrm{nC}$ for the delay $\sim 5.7 \mathrm{~mm}$, from 10 to $12 \mathrm{nC}$ for the delay $\sim 10.7 \mathrm{~mm}$, and from 13 to $15 \mathrm{nC}$ for the delay $\sim 21.7 \mathrm{~mm}$. For each delay, multiple shots were recorded on the spectrometer screen; typical information obtained on each shot include the energy gain/loss received by electrons and the horizontal deflection (kick) received by electrons. The changes in energy are read by taking a sum 
TABLE III. Comparison of observed energy gain/loss values with the model predictions.

\begin{tabular}{lccc}
\hline \hline Delay $(\mathrm{mm})$ & 5.7 & 10.7 & 21.7 \\
\hline Expected maximum gain $(\mathrm{MeV} / \mathrm{m})$ & 6.4 & 8 & 9 \\
Observed maximum gain $(\mathrm{MeV} / \mathrm{m})$ & 5.6 & 6.73 & 11.7 \\
Expected minimum gain, or loss $(\mathrm{MeV} / \mathrm{m})$ & -4.97 (loss) & -2.92 (loss) & +6.0 (gain) \\
Observed minimum gain, or loss $(\mathrm{MeV} / \mathrm{m})$ & -5.6 (loss) & -2.9 (loss) & +6.5 (gain) \\
\hline \hline
\end{tabular}

of projections of the image vertical slices on the screen; the changes in the horizontal position are read by taking a sum of projections of the image horizontal slices on the screen $[16,18]$. Every time, the background level is subtracted, and for convenience the resulting distributions are normalized to have their maxima equal to unity. The energy slit helps to narrow the energy value; being positioned horizontally, the energy slit, however, does not affect the readouts to infer the horizontal deflection of the bunch, which is later processed to obtain the value of the responsible horizontal deflecting force [17].

With the delay of $\sim 5.7 \mathrm{~mm}$ the typical energy gain was up to $50-100 \mathrm{keV}$ and the energy loss was up to 90-100 keV; on average the energy changed by $\sim 0 \mathrm{keV}$. For delay $\sim 11 \mathrm{~mm}$, the jitter of 50-60 keV and the energy slit error $77 \mathrm{keV}$ required some corrections. Taking these into account, the energy loss was up to $65 \mathrm{keV}$, while the energy gain was in the range $65-150 \mathrm{keV}$; the average energy change was $\sim 50 \mathrm{keV}$. For delay $\sim 22 \mathrm{~mm}$, the jitter of $40-50 \mathrm{keV}$ and the energy slit error $77-154 \mathrm{keV}$ required some corrections. The energy gain was up to $350 \mathrm{keV}$; the average energy change was $\sim 170-220 \mathrm{keV}$.

To compare with the theory model predictions, it is convenient to take into account the measured drive charges, and renormalize the gains/losses to $50 \mathrm{nC}$ of drive charge; this normalization is a linear one under an assumption that the drive bunch sizes do not vary when its charge changes. Table III presents the comparison.

The top plot [plot (a)] in Figs. 12-14 presents two theoretical $F_{z}$ curves computed for the drive bunch $x$ shifted by 2 and $3 \mathrm{~mm}$, respectively, where the outermost arrow heads indicate the observed values (renormalized to $50 \mathrm{nC}$ ). A good agreement can be seen in all cases. With regards to the delay $\sim 5.7 \mathrm{~mm}$, it should be noted that some shots demonstrated the gain only as high as $2.8 \mathrm{MeV} / \mathrm{m}$ (renormalized to $50 \mathrm{nC}$ of the drive charge); this is, however, within the expectations [see Fig. 12(a), middle arrow]. At the delays $\sim 10.7$ and $21.7 \mathrm{~mm}$, the average expected gain values are 2.92 and $8.5 \mathrm{MeV} / \mathrm{m}$; the observed values [see, e.g., Figs. 13(a) and 14(a); pointed at by the middle arrow heads] were 2.24-2.91 and $\sim 8.46 \mathrm{MeV} / \mathrm{m}$ (again renormalized here to $50 \mathrm{nC}$ ), and again are in a good agreement with the expectations.

To study the changes in energy distribution caused by the interaction between the test bunch and the wakefields set up by the preceding drive bunch, the method described in Appendix A 1 is used. The middle plot [plot (b)] in
Figs. 12-14 presents a typical observed distribution when the test bunch does not interact (green) and interacts with the wakefields (red). These compares favorably with the simulated behaviors presented for each delay (with the
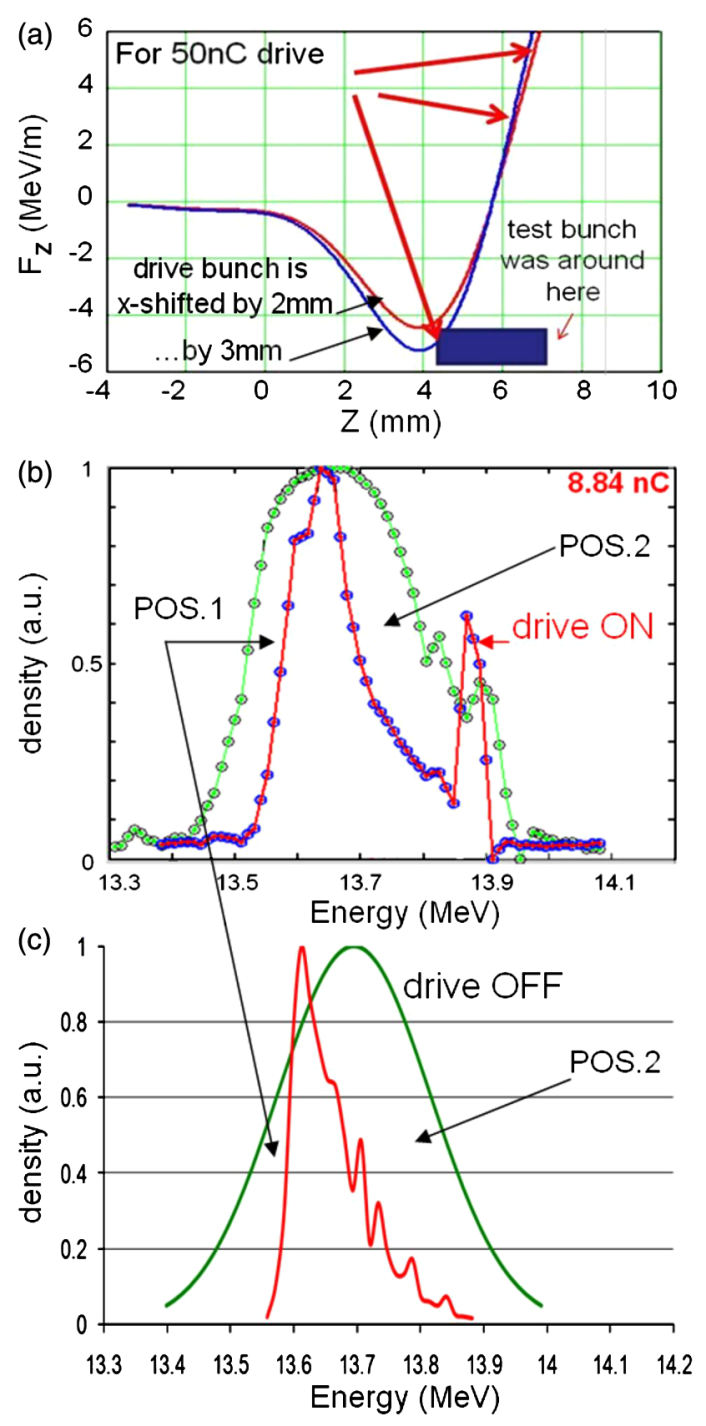

FIG. 12. (a) Simulations of $F_{z}$ for the test channel [values are renormalized to $50 \mathrm{nC}$ (see explanations in the text)] for the delay $5.7 \mathrm{~mm}$. (b) Typical energy distribution (normalized to 1) observed in $80 \%-85 \%$ of shots. (c) CST MICROWAVE STUDIO simulations to predict changes in the energy distribution [for case \#1 in Table V, and the drive bunch is shifted off the center of its channel by $2 \mathrm{~mm}$ (toward the test channel)]. 

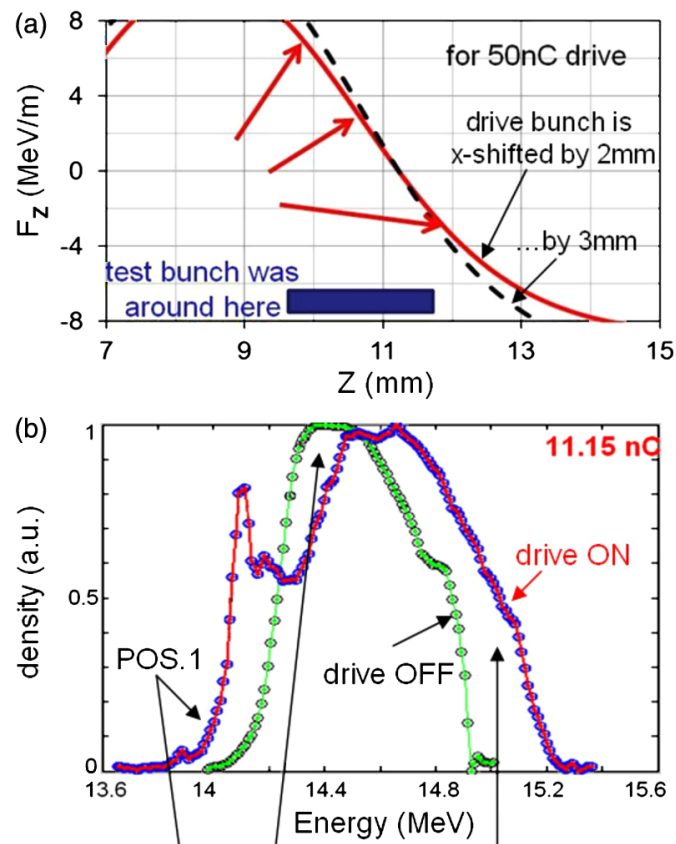

(c)

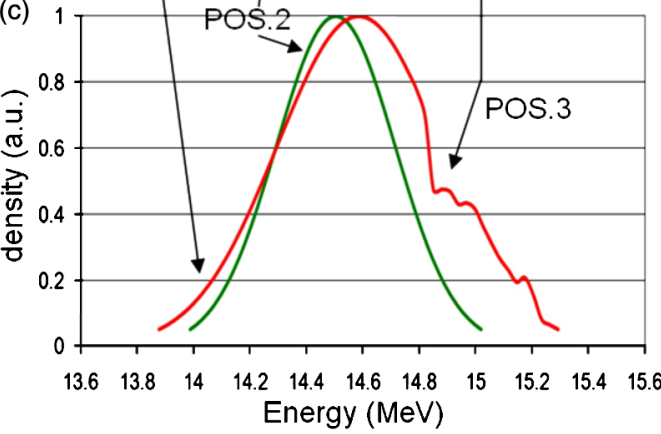

FIG. 13. (a) Simulations of $F_{z}$ for the test channel [values are renormalized to $50 \mathrm{nC}$ (see explanations in the text)] for the delay $10.7 \mathrm{~mm}$. (b) Typical energy distribution (observed in $80 \%$ of shots; normalized to 1). (c) CST MICROWAVE STUDIO simulations to predict changes in the energy distribution [for case \#2 in Table V, and the drive bunch being shifted off the center of its respective channel by $2 \mathrm{~mm}$ (toward the test channel)].

corresponding drive charge) by the bottom plot [plot (c)] in Figs. 12-14.

For instance, in Fig. 12, the slope of final energy distribution (drive $\mathrm{ON}$ ) in position 1 is sharper than the slope in position 2 in both experiment and simulations. Also, the final energy distribution - in both experiment and simulations-shrinks inward of the initial energy distribution (drive OFF).

In Fig. 13, in position 1 the final energy distribution (drive $\mathrm{ON}$ ) moves to the left in both top and bottom figures as compared to the initial distribution (drive OFF). In position 2 the final energy distribution moves "inward" of the initial one, again on both plots. In position 3 , the behavior is again the same.

In Fig. 14, the final distribution (drive ON) is shown when the drive bunch is shifted off the center of its (a)

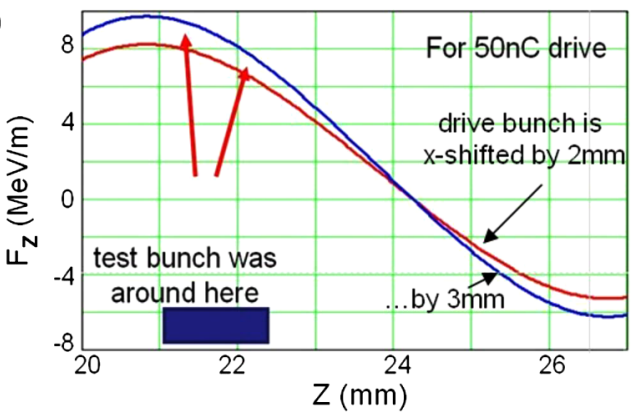

(b)

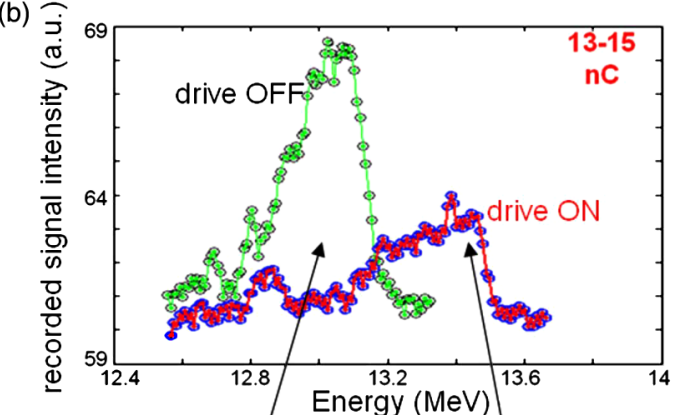

(c)

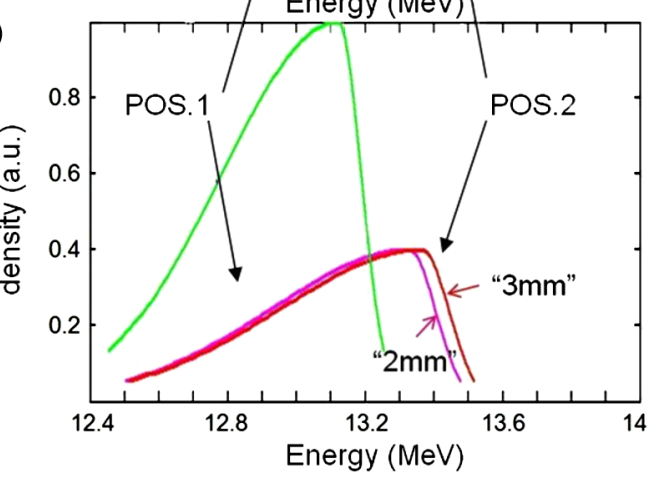

FIG. 14. Simulations of $F_{z}$ for the test channel [values are renormalized to $50 \mathrm{nC}$ (see explanations in the text)] for the delay $21.7 \mathrm{~mm}$. (b) Typical energy distribution (observed in $80 \%$ of shots; raw intensity data are shown). (c) Simulations to predict changes in the energy distribution (for case \#3 in Table V).

respective channel by 2 and $3 \mathrm{~mm}$ (curves are pointed at by " $2 \mathrm{~mm}$ " and " $3 \mathrm{~mm}$," respectively, in the bottom plot). In position 1 and position 2 , the evolution of final energy distribution is the same in both top and bottom figures as compared to the initial distribution (drive OFF). Note that some particle losses do occur in this case.

\section{CHANGES IN BUNCH HORIZONTAL DISTRIBUTION AFTER ACCELERATION: EXPERIMENTAL RESULTS VS MODEL PREDICTIONS}

Here again, the data were collected for the same three different delays between the drive bunch and the test bunch, namely $\sim 5.7, \sim 10.7$, and $\sim 21.7 \mathrm{~mm}$. The data acquisition and processing procedures have already been described in the former section. 
TABLE IV. Comparison of observed $F_{x}$ values with the model predictions.

\begin{tabular}{lccc}
\hline \hline Delay $(\mathrm{mm})$ & 5.7 & 10.7 & 21.7 \\
\hline Expected maximum $F_{x}(\mathrm{MeV} / \mathrm{m})$ & +5.38 & -4.4 & +8.7 \\
Observed maximum $F_{x}(\mathrm{MeV} / \mathrm{m})$ & +5.12 & -3.6 & +7.35 \\
Expected minimum $F_{x}(\mathrm{MeV} / \mathrm{m})$ & +4 & -1.45 & +4.4 \\
Observed minimum $F_{x}(\mathrm{MeV} / \mathrm{m})$ & +4.65 (typical) & -1.72 & +4.5 \\
\hline \hline
\end{tabular}

With the delay $\sim 5.7 \mathrm{~mm}$, the horizontal kick that led to the shift as shown, e.g., in Fig. 15(b) was about $6.18-6.8 \mathrm{mrad}$. For delay $\sim 10.7 \mathrm{~mm}$, the horizontal kick that led to the typical shifts presented in, e.g., Fig. 16(b) was ranging from -2.45 to $-5.2 \mathrm{mrad}$; in average it was $-3.9 \mathrm{mrad}$. For delay $\sim 21.7 \mathrm{~mm}$, the horizontal kick was inferred to be about $+12.2 \mathrm{mrad}$ in average.
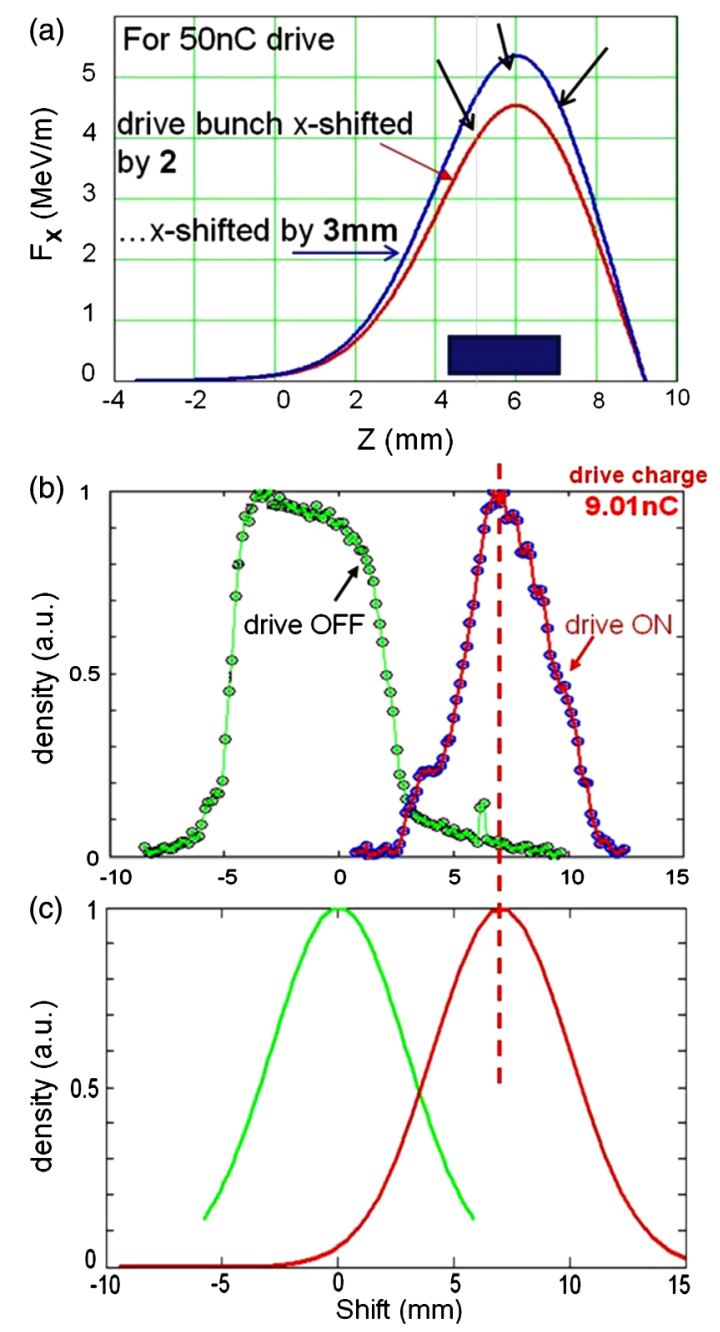

FIG. 15. (a) Simulations of $F_{x}$ for the test channel. (b) Typical bunch horizontal distribution (observed in $80 \%-85 \%$ of shots, normalized to 1) for the delay was $5.7 \mathrm{~mm}$. (c) Simulations that predict changes in the horizontal distribution [for case \#1 in Tables V and VI, for the drive bunch being shifted off the center of its respective channel by $2 \mathrm{~mm}$ (toward the test channel)].
The aforementioned kick is the result of the $F_{x}$ force acting on the test bunch, and its value can be inferred from the aforementioned kick values. To compare with the theory model predictions, it is again convenient to take into account the measured drive charges, and renormalize the $F_{x}$ to $50 \mathrm{nC}$ of drive charge; this normalization is a linear one under an assumption that the drive bunch sizes do not vary when its charge changes. Table IV presents the comparison.
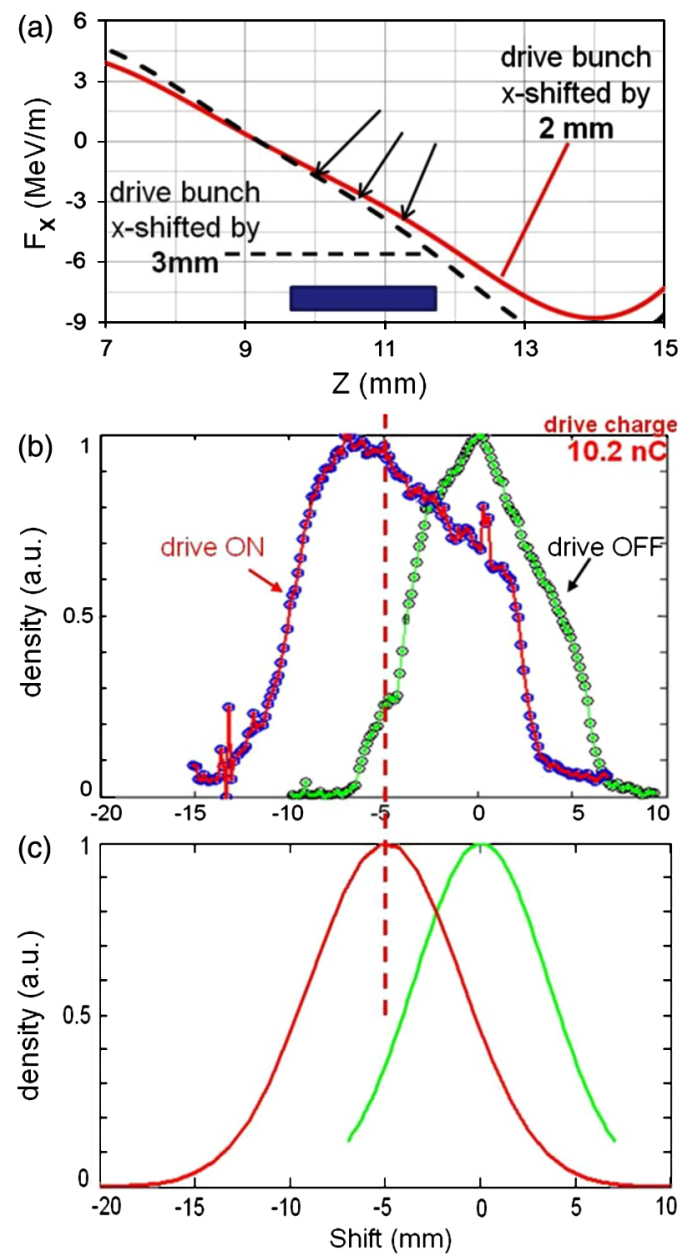

FIG. 16. (a) Simulations of $F_{x}$ for the test channel. (b) Typical bunch horizontal distribution (observed in $80 \%$ of shots, normalized to 1) when the delay was $10.7 \mathrm{~mm}$. (c) Predicted changes in horizontal distribution [case \#2 in Tables V and VI with the drive bunch being shifted off the center of its respective channel by $2 \mathrm{~mm}$ (toward the test channel)]. 
In addition to Table IV, the top plot [plot (a)] in Figs. 15-17 presents two theoretical $F_{x}$ curves computed for the drive bunch $x$ shifted by 2 and $3 \mathrm{~mm}$, respectively, where the arrow heads indicate some of the observed values (renormalized to $50 \mathrm{nC}$ ). A good agreement can be seen in all cases. With regard to Fig. 15, it should be noted that even the value of $\sim 4 \mathrm{MeV} / \mathrm{m}$ was observed on occasions (leftmost arrow) as Table IV predicts. In Fig. 16(a), the middle arrow shows the observed average value $(-2.8 \mathrm{MeV} / \mathrm{m})$, which appears to be in a favorable agreement with expectations. In Fig. 17, two arrows point at the observed values 5.6 and $6.1 \mathrm{MeV} / \mathrm{m}$, which are also the expected $F_{X}$ value that should cause the average kick of $\sim 12.2 \mathrm{mrad}$ (difference 5.6 vs 6.1 comes from the difference in drive bunch charge).

(a)

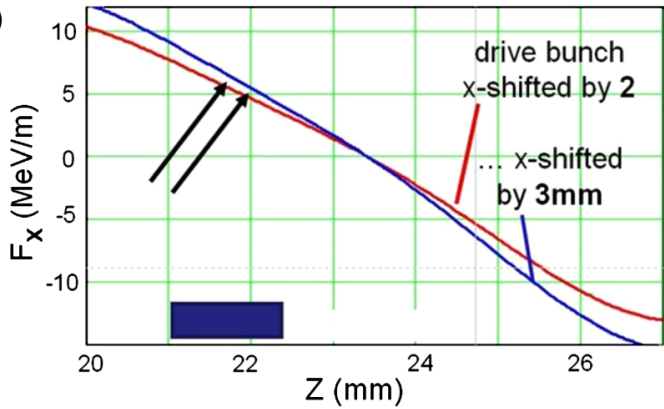

(b)

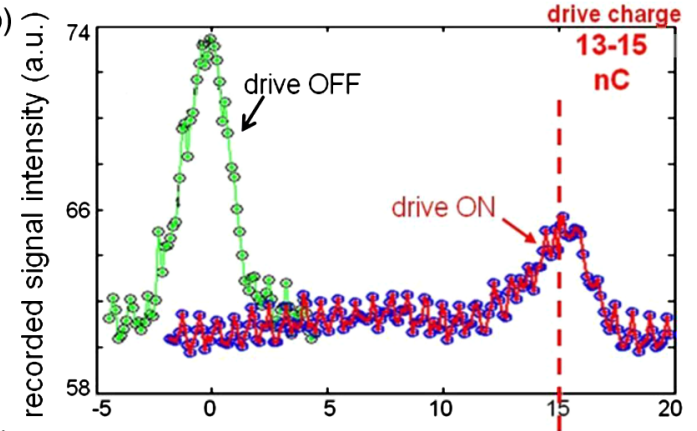

(c)

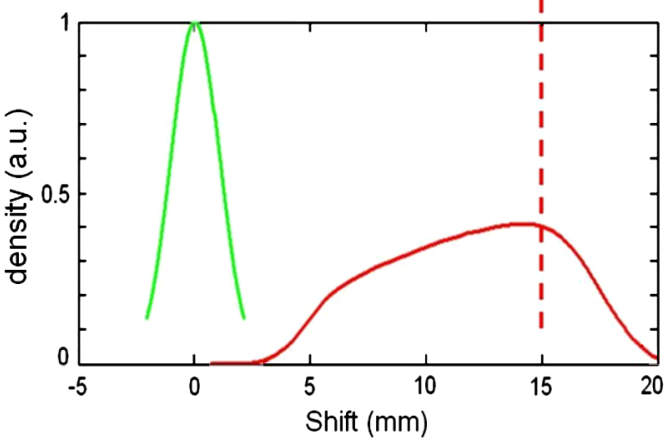

FIG. 17. (a) Simulations of $F_{x}$ for the test channel. (b) Typical bunch horizontal distribution (observed in $80 \%$ of shots) when the delay was $21.7 \mathrm{~mm}$; note that these are examples of "raw" distributions. (c) Changes in horizontal distribution [for case \#3 in Tables V and VI with the drive bunch being shifted off the center of its respective channel by $2 \mathrm{~mm}$ (toward the test channel)].
To further study the changes in horizontal distribution (and $x$ kicks) caused by the interaction between the test bunch and the wakefields set up by the preceding drive bunch, the method described in Appendix A 2 is used. The middle plot [plot (b)] in Figs. 15-17 presents a typically observed distribution when the test bunch does not interact (green) and interacts with the wakefields (red). These compare favorably with the simulated behaviors presented for each delay (with the corresponding drive charge and the initial FWHM chosen to be the same as the initial observed FWHM) by the bottom plot [plot (c)].

In Fig. 15, one observes the same amount of average $x$ kick for the accelerated test bunch (curves marked by "drive ON").

In Fig. 16, one observes again nearly the same amount of average $x$ kick for the accelerated test bunch (curves marked by "drive ON"), and also that in both experiment and theory FWHM appears to remain the same before and after acceleration.

Finally in Fig. 17, one sees nearly the same amount of $x$ kick for the accelerated test bunch (curves marked by "drive ON") where the distribution has its peak, and also that in both experiment and theory, the left slope is far more elongated than the right slope in the final distribution.

\section{CONCLUSIONS}

To the best of our knowledge, the experiments described in this paper are the first in which a two-channel, dielectriclined, rectangular, wakefield structure has been tested. It is found that the experimental data are consistent with the theory model predictions. The theory model includes the wakefield simulations using the CST STUDIO, together with reasonable assumptions regarding the input distribution of bunch particle energies and positions.

Of course, introducing a narrower test bunch at particular values of the delay would secure both high acceleration and low deflection. Nevertheless, the presence of deflection in a rectangular DWFA is an intrinsic feature [17] that is a consequence of its lack of symmetry. A more promising choice for accelerator application should be a coaxial structure where symmetry can cure this unwanted deflection $[12,15]$. The rectangular version-studied [14,16-18] and developed $[14,16]$ by our group-nonetheless delivers a useful example that is very well suited to check theory and our understanding of how wakefields in a two-channel structure manifest themselves.

Finally, we note that the aforementioned practical difficulties to operate a two-bunch scheme as described in Sec. II, where both bunches are produced at the same rf photocathode with one of them delayed and propagating off the beam-line axis, suggest that two-bunch noncollinear structures are better tested at a facility that is equipped with two guns to produce, and two separate beam lines to manipulate, the bunches independently. 


\section{ACKNOWLEDGMENTS}

The authors would like to acknowledge the financial support of U.S. Department of Energy (Office of High Energy Physics), and also express many thanks to A. Didenko (Omega-P contractor) for his excellent mechanical design for the rectangular DWFA module and related hardware, and to J. Zmuda, M. Lien, and R. Konecny (Argonne National Laboratory) for their help during the installations, operation, and especially for refurbishing the modulator that enabled us to have excellent operational stability - much needed for this experiment which required two time-delayed bunches that originate at the same photocathode with the second one produced and delivered off the beam-line axis.

\section{APPENDIX A}

\section{Changes in bunch energy after acceleration}

To study the changes in energy distribution caused by the interaction between the test bunch and the wakefields set up by the preceding drive bunch, it is assumed here and further on that the initial longitudinal distribution in the test bunch resembles a Gaussian one, however, with the head possibly different from the tail. In that case, when a bunch with a small charge is produced by an rf photocathode gun, a validated model [30-32] is

$$
\begin{array}{ll}
n_{\text {ini }}(z)=n_{0} \exp \left(-\frac{\left(z-Z_{\text {test }}\right)^{2}}{2 \sigma_{z, \text { head }}^{2}}\right), & \text { if } z \leq Z_{\text {test }}(\text { A } 1 \mathrm{a}) \\
n_{\text {ini }}(z)=n_{0} \exp \left(-\frac{\left(z-Z_{\text {test }}\right)^{2}}{2 \sigma_{z, \text { tail }}^{2}}\right), & \text { if } z>Z_{\text {test }} \text { (A1b) }
\end{array}
$$

where $n_{\text {ini }}(z)$ is the initial particle density, $z$ is the initial longitudinal coordinate of the test particle relative to the drive bunch (center of bunch is located at $z=0$, positive coordinate $z$ corresponds to the location after the bunch center, negative coordinate $z$ corresponds to the location before the bunch center), $Z_{\text {test }}$ is the longitudinal position (delay) of the test bunch center relative to the drive bunch, $\sigma_{z \text {,head }}$ and $\sigma_{z \text {,tail }}$ are the rms- length of the bunch head and its tail, and $n_{o}$ is an appropriate normalization constant. The values of delay, $Z_{\text {test }}$, and $\sigma_{z \text {,head }}$ and $\sigma_{z \text {,tail }}$ as either directly measured in experiment or inferred from processing the experimental data are listed in Table V.

The correlation between the test particle's initial position and its initial energy is important to explain the behavior already presented. This information is not available, so here we must make an assumption about this correlation; we take it to be approximated by a linear function:

$$
\begin{aligned}
E_{\text {ini }}(z) & =E_{\text {test }}-\left(z-Z_{\text {test }}\right) \frac{\sigma_{E}}{\sigma_{Z}} \\
\text { with } \sigma_{Z} & =0.5\left(\sigma_{z \text {,head }}+\sigma_{z, \text { tail }}\right),
\end{aligned}
$$

where $E_{\text {ini }}(z)$ is the test particle initial energy, $E_{\text {test }}$ is an average initial energy of the test bunch known from the experiment, and $\sigma_{E}$ is the rms-energy spread of the test bunch (also known from the experimental curves, see, e.g., Figs. 12-14). $E_{\text {test }}$ and $\sigma_{E}$ are listed in Table V. $\sigma_{z}$ is selected to be computed as indicated below.

The particle initial coordinate $z$ in Eqs. (A1) and (A2) is a parameter; thus, one can plot $n_{\text {ini }}(z)$ vs $E_{\text {ini }}(z)$ and adjust $\sigma_{z \text {,head }}$ and $\sigma_{z \text {,tail }}$ to accurately model the observed initial energy distribution as seen in Figs. 12, 13, and 14(b). These values are presented in Table V. The fields/forces that dictate the particle dynamics are known from the IVF codes and the CST STUDIO simulations (see Fig. 18).

After the equations of motion are integrated over the apparatus length, $L$, the final particle position (relative to the drive bunch) and final energy are

$$
\begin{aligned}
z_{\text {fin }} & =\tilde{Z}\left(E_{\text {ini }}, z\right) \\
n_{\text {fin }}\left(z_{\text {fin }}\right) & =n_{\text {ini }}(z)-\text { PPL } \\
E_{\text {fin }} & =\tilde{E}\left(E_{\text {ini }}, z\right),
\end{aligned}
$$

where (as before) $z$ is the initial particle coordinate (relative to the drive bunch), $E_{\text {ini }}$ is the initial energy, $z_{\text {fin }}$ is the final coordinate, $\tilde{Z}(\ldots)$ is a function that represents the dependence of $z_{\text {fin }}$ on $E_{\text {ini }}$ and $z$, and is obtained after numerical integration; $n_{\text {fin }}$ is the particle density at $z_{\text {fin }}$, with PPL representing possible particle losses because some particles may hit the walls of the test channel, $E_{\text {fin }}$ is the final energy, and $\tilde{E}(\ldots)$ is a function that represents the dependence of $E_{\text {fin }}$ on $E_{\text {ini }}$ and $z$, and is also obtained after numerical integration.

Noting that $z$ enters as a parameter in Eq. (A3), and that Eq. (A2) provides the correlation between $E_{\text {ini }}$ and $z$, one can mutually exclude $z$ from Eqs. (A3b) and (A3c), and then plot $n_{\text {fin }}$ vs $E_{\text {fin }}$ to obtain the final energy distribution.

The aforementioned recipes produce results for different delays ( $\sim 6, \sim 11$, and $\sim 22 \mathrm{~mm}$ ) as have been presented in Figs. 12-14, with a very good agreement seen in all cases.

TABLE V. Test bunch parameters.

\begin{tabular}{lccc}
\hline \hline Case \# & 1 & 2 & 3 \\
\hline$Z_{\text {test }}(\mathrm{mm})$, delay between drive and test bunches & 5.7 & 10.7 & 21.7 \\
$E_{\text {test }}(\mathrm{MeV})$ & 13.7 & 14.5 & 13.12 \\
$\sigma_{z, \text { head }} / \sigma_{z, \text { tail }}(\mathrm{mm})$ & $0.6 / 0.6$ & $0.6 / 0.6$ & $0.2 / 1.0$ \\
$\sigma_{E}(\mathrm{MeV})$ & 0.12 & 0.21 & 0.2 \\
\hline \hline
\end{tabular}




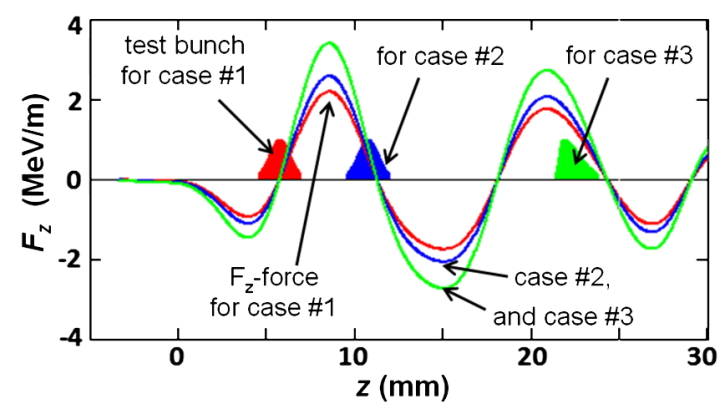

FIG. 18. $\quad F_{z}(z)$-force acting on the test bunch (traveling behind the drive bunch at locations marked as case 1 , or 2 or 3 ) is shown here computed for $9 \mathrm{nC}$ of the drive charge (case \#1), $10.6 \mathrm{nC}$ of the drive charge (case \#2), and $14 \mathrm{nC}$ (case \#3). The drive bunch is off the center of its respective channel by $3 \mathrm{~mm}$ toward the test channel. Accordingly, for each case the test bunch position and distribution is different, as indicated.

In particular, in a zero-order approximation, one may assume the absence of particle slippage and transverse motion (because of the short apparatus length, $L$ ), and Eq. (A3) becomes

$$
z_{\text {fin }}=z \quad n_{\text {fin }}\left(z_{\text {fin }}\right)=n_{\text {ini }}(z) \text {, and } E_{\text {fin }}=E_{\text {ini }}(z)+F_{z}(z) L,
$$

where $F_{z}$ is presented in Fig. 18.

In this simplified scenario, the final distribution is, obviously, a plot of

$$
n_{\mathrm{ini}}(z) \text { vs } E_{\text {ini }}(z)+F_{z}(z) L .
$$

\section{Bunch $X$ deflection}

To study the change in horizontal velocity distribution that results from the transverse wakefield force, we use the following zero-order approximation for the initial test bunch distribution: (1) the bunch is relatively compact horizontally and vertically, that is $\sigma_{x}=\sigma_{y}=0$; (2) the velocity spread in the $y$ direction is ignored; (3) the velocity spread in the $x$ direction is taken into account in the particle density distribution function as

$$
N_{\text {ini }}\left(z, \alpha_{x}\right)=n_{0} n_{\text {ini }}(z) \exp \left(-\frac{\alpha_{x}^{2}}{2 \sigma_{\alpha, x}^{2}}\right)
$$

where $\alpha_{x}=V_{x} / c$ is the $x$ velocity represented as an angular value (rad) with $V_{x}$ being the horizontal transverse velocity measured in $\mathrm{m} / \mathrm{sec} ; \sigma_{\alpha, x}=\sigma_{v, x} / c$ is the horizontal rms spread represented as an angular value (rad), and $c$ is the speed of light ( $\sim$ the longitudinal velocity with which $13-14 \mathrm{MeV}$ electrons move); $n_{o}$ is an appropriate normalizing constant; $n_{\text {ini }}(z)$ is given by Eq. (A1) in Appendix A.

Because initially $z$ and $\alpha_{x}$ have no correlation between each other, the distribution as a function of the $x$ velocity is simply
TABLE VI. Test bunch parameters (see also Table V).

\begin{tabular}{llll}
\hline \hline Case \# & 1 & \multicolumn{1}{c}{2} & 3 \\
\hline $1230 \mathrm{~mm} \cdot \sigma_{\alpha, x}$ & $2.91 \mathrm{~mm}$ & $3.5 \mathrm{~mm}$ & $1.05 \mathrm{~mm}$ \\
$\sigma_{\alpha, x}$ & $2.36 \mathrm{mrad}$ & $2.84 \mathrm{mrad}$ & $0.85 \mathrm{mrad}$ \\
\hline \hline
\end{tabular}

$$
N_{\text {ini }}\left(\alpha_{x}\right)=n_{1} \exp \left(-\frac{\alpha_{x}^{2}}{2 \sigma_{\alpha, x}^{2}}\right)
$$

where $n_{1}$ is an appropriate normalizing constant.

The values of $\sigma_{\alpha, x}$ are found by analyzing the initial horizontal particle distributions available from the experiment. If the distributions are assumed to resemble Gaussian ones, the rms values $(\mathrm{mm})$ seen on the spectrometer screen are merely $1230 \mathrm{~mm} \cdot \sigma_{\alpha, x}$ because the distance between the apparatus and the screen was/is $1230 \mathrm{~mm}$. Practically, it is easier to measure the FWHM, and then cast this to rms values remembering that $\sigma_{\alpha, x}=$ FWHM/2.35 for any Gaussian distribution. The rms values are listed in Table VI.

The angle, $\alpha_{x, \text { fin }}$, when the electron exits the apparatus is given by (again, we use the zero-order approximation in our model)

$$
\alpha_{x, \text { fin }}=\alpha_{x}+L \frac{F_{x}(z)}{E_{\text {test }}},
$$

where $\alpha_{x}$ is the initial angle, $L=100 \mathrm{~mm}$ is the DWFA apparatus length, $F_{x}(\mathrm{MeV} / \mathrm{m})$ is computed by CST STUDIO and is given in Fig. 19, $z$ is the initial position of the electron within the bunch, and $E_{\text {test }}$ is the test bunch average energy. Here we assume that the slippage between

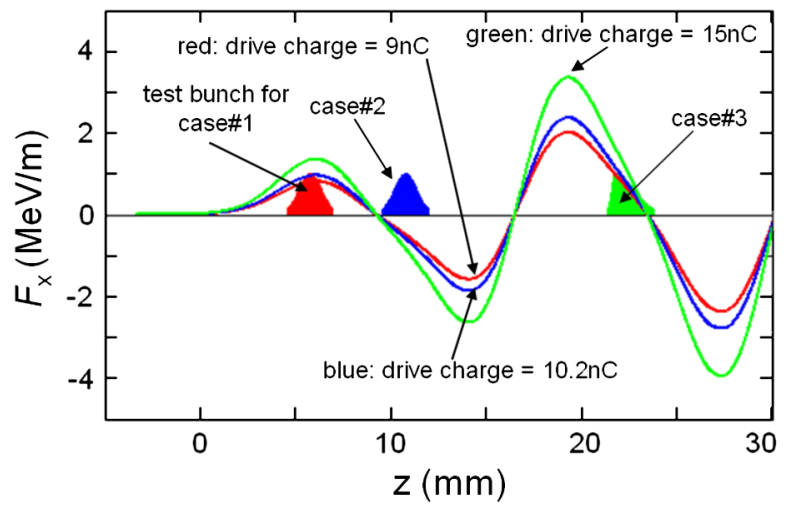

FIG. 19. $\quad F_{x}(z)$ as computed by IVF codes dictates the final velocity spread and distribution; note bunches and wakefields move from right to left, toward lesser $z$ values. $F_{x}(z)$-force acting on the test bunch (traveling behind the drive bunch at locations marked as case 1, or 2 or 3 ) is shown here computed for $9 \mathrm{nC}$ of the drive charge (case \#1), $10.2 \mathrm{nC}$ of the drive charge (case \#2), and $15 \mathrm{nC}$ (case \#3). The drive bunch is off the center of its respective channel by $2 \mathrm{~mm}$ toward the test channel. For each case the test bunch position and distribution is different, as indicated. 
the electron and the wakefield is negligible, and energy spread is small compared to $E_{\text {test }}$ (as it has been already confirmed by material presented before in Sec. IV).

Thus, in this no-slippage approximation, one has $\alpha_{x}=$ $\alpha_{x, \text { fin }}-L F_{x}(z) / E_{\text {test }}$ that allows one to trace particles to the place of their origin where the distribution is known. Hence, the final distribution becomes

$N_{\text {fin }}\left(z, \alpha_{x, \text { fin }}\right)=n_{\text {of }} n_{\text {ini }}(z) \exp \left(-\frac{\left[\alpha_{x, \text { fin }}-L F_{x}(z) / E_{\text {test }}\right]^{2}}{2 \sigma_{\alpha, x}^{2}}\right)$,

where all the values and parameters already have been identified on the previous pages (and $n_{\text {of }}$ is an appropriate normalizing constant).

The final distribution in the $x$-velocity space is obtained by integrating the above formula over all $z$ values (where the beam particles can be found); the integration is a trivial procedure when one employs MATHCAD, MATHEMATICA, or a custom $\mathrm{C}++/$ Fortran program. The final distribution is

$$
\begin{aligned}
N_{\text {fin }}\left(\alpha_{x, \text { fin }}\right)= & n_{1 f} \int_{z} d z \cdot n_{\text {ini }}(z) \\
& \times \exp \left(-\frac{\left[\alpha_{x, \text { fin }}-L F_{x}(z) / E_{\text {test }}\right]^{2}}{2 \sigma_{\alpha, x}^{2}}\right),
\end{aligned}
$$

where $n_{1 f}$ is an appropriate normalizing constant. Note that both $n_{\text {ini }}(z)$ and $F_{x}(z)$ depend on $z$, and must be integrated en masse.

Using Eqs. (A5) and (A7), one can compare the initial and final distributions. When graphing them, it is convenient to plot

$$
\begin{aligned}
& N_{\text {ini }}\left(\alpha_{x}\right) \text { vs } \alpha_{x} \cdot 1230 \mathrm{~mm} \\
& N_{\text {fin }}\left(\alpha_{x, \text { fin }}\right) \text { vs } \alpha_{x, \text { fin }} \cdot 1230 \mathrm{~mm}
\end{aligned}
$$

because $\alpha_{x} \cdot 1230 \mathrm{~mm}$ and $\alpha_{x, \text { fin }} \cdot 1230 \mathrm{~mm}$ are the shifts $(\mathrm{mm})$ one measures directly on the spectrometer screen along its $X$ axis [see for instance Figs. 15, 16, and 17(b)]. As it has been demonstrated, the theory predictions are well confirmed by the experimental observations.

[1] G. V. Sotnikov and T. C. Marshall, Phys. Rev. ST Accel. Beams 14, 031302 (2011).

[2] A. Kanareykin, S. P. Antipov, J. B. Butler, C.-J. Jing, P. Schoessow, and W. Gai, in Proceedings of the 2011 Particle Accelerator Conference, New York, 2011, edited by T. Satotaga and K. Brown (IEEE, New York, 2011), p. 319.

[3] J. Rosenzweig et al., High Frequency High Gradient Dielectric Wakefield Acceleration Experiment at SLAC and BNL, AIP Conf. Proc. No. 1299, edited by S. H. Gold and G.S. Nusinovich (AIP, New York, 2010), p. 364, and references therein.

[4] Liling Xiao, Wei Gai, and Xiang Sun, Phys. Rev. E 65, 016505 (2001).
[5] J. G. Power, Wei Gai, and Paul Schoessow, Phys. Rev. E 60, 6061 (1999).

[6] J. G. Power, M.E. Conde, W. Gai, R. Konecny, P. Schoessow, and A. D. Kanareykin, Phys. Rev. ST Accel. Beams 3, 101302 (2000).

[7] W. Gai, A. D. Kanareykin, A. L. Kustov, and J. Simpson, Phys. Rev. E 55, 3481 (1997).

[8] C. Jing, A. Kanareykin, J. G. Power, M. Conde, W. Liu, S. Antipov, P. Schoessow, and W. Gai, Phys. Rev. Lett. 106, 164802 (2011).

[9] C. Jing, J. G. Power, M. Conde, W. Liu, Z. Yusof, A. Kanareykin, and W. Gai, Phys. Rev. ST Accel. Beams 14, 021302 (2011).

[10] C. Jing et al., Recent Experiments on Wakefield Transformer Ratio Enhancement at AWA, AIP Conf. Proc. No. 1299, edited by S.H. Gold and G.S. Nusinovich (AIP, New York, 2010), p. 348.

[11] A. Kanareykin et al., Development of a Ferroelectric Based Tunable DLA Structure, edited by C.B. Schroeder, W. Leemans, and E. Esarey, AIP Conf. Proc. No. 1086 (AIP, New York, 2009), p. 386.

[12] T. C. Marshall, G. V. Sotnikov, and J. L. Hirshfield, A THz Coaxial Two-Channel Dielectric Wakefield Structure for High Gradient Acceleration, edited by S.H. Gold and G. S. Nusinovich, AIP Conf. Proc. No. 1299 (AIP, New York, 2010), p. 336.

[13] G. V. Sotnikov, T.C. Marshall, J.L. Hirshfield, and S. V. Shchelkunov, Accelerated Bunch Stability in a Coaxial Dielectric Wakefield Structure When its Symmetry is Broken, edited by S.H. Gold and G. S. Nusinovich, AIP Conf. Proc. No. 1299 (AIP, New York, 2010), p. 342.

[14] T. C. Marshall, G. V. Sotnikov, S. V. Shchelkunov, and J. L. Hirshfield, Analysis of a Symmetric Terahertz DielectricLined Rectangular Structure for High Gradient Acceleration, edited by C. B. Schroeder, W. Leemans, and E. Esarey, AIP Conf. Proc. No. 1086 (AIP, New York, 2009), p. 421.

[15] G. V. Sotnikov, T. C. Marshall, and J. L. Hirshfield, Phys. Rev. ST Accel. Beams 12, 061302 (2009).

[16] S. V. Shchelkunov, M. A. LaPointe, M. E. Conde, W. Gai, J. G. Power, Z. M. Yusof, J. L. Hirshfield, T. C. Marshall, D. Mihalcea, and G. V. Sotnikov, in Proceedings of the 2011 Particle Accelerator Conference, New York, 2011 (Ref. [2]), p. 298 [http://accelconf.web.cern.ch/ AccelConf/PAC2011/papers/mop107.pdf].

[17] J. L. Hirshfield, T.C. Marshall, S. V. Shchelkunov, and G. V. Sotnikov, in Proceedings of the 23rd Particle Accelerator Conference (PAC2009), Vancouver, BC, Canada, 2009, edited by M. Comyn (IEEE, Piscataway, NJ, 2009), p. 4267.

[18] G. V. Sotnikov, T.C. Marshall, S. V. Shchelkunov, A. Didenko, and J. L. Hirshfield, Two-Channel Rectangular Dielectric Wake Field Accelerator Structure Experiment, edited by C. B Schroeder, W. Leemans, and E. Esarey, AIP Conf. Proc. No. 1086 (AIP, New York, 2009), p. 415.

[19] S. V. Shchelkunov, T. C. Marshall, J. L. Hirshfield, M. A. Babzien, and M. A. LaPointe, Phys. Rev. ST Accel. Beams 9, 011301 (2006).

[20] S. V. Shchelkunov, T. C. Marshall, J. L. Hirshfield, M. A. Babzien, and M.A. LaPointe, in Proceedings of the 
Particle Accelerator Conference, Knoxville, 2005, edited by C. Horak (IEEE, Piscataway, NJ, 2005), p. 3609.

[21] Wanming Liu and Wei Gai, Phys. Rev. ST Accel. Beams 12, 051301 (2009).

[22] Chunguang Jing, Wanming Liu, Liling Xiao, Wei Gai, P. Schoessow, and Thomas Wong, Phys. Rev. E 68, 016502 (2003).

[23] S. Antipov et al., Diamond-Based Dielectric Loaded Accelerating Structures, edited by and, AIP Conf. Proc. No. 1299 (AIP, New York, 2010), p. 359.

[24] C. Jing et al., Development of Transverse Modes Damped DLA Structure, edited by C. B. Schroeder, and E. Esarey, AIP Conf. Proc. No. 1086 (AIP, New York, 2009), p. 433.

[25] C. Jing, A. Kanareykin, J. G. Power, M. Conde, Z. Yusof, P. Schoessow, and W. Gai, Phys. Rev. Lett. 98, 144801 (2007).

[26] M. E. Conde, D. S. Doran, W. Gai, R. Konecny, W. Liu, J. G. Power, Z.M. Yusof, S.P. Antipov, C.-J. Jing, and E.E. Wisniewski, in Proceedings of the 2011
Particle Accelerator Conference, New York, 2011 (Ref. [2]), p. 115.

[27] M. Conde, "Argonne Wakefield Accelerator Facility (AWA) Upgrades and Future Goals" [https://twindico .hep.anl.gov/indico/getFile.py/access? contribId=29 $\&$ resId=0\&materialId $=$ slides $\&$ confId $=428 \# 256,1$, AWA Facility Upgrade].

[28] C. Wang and J. L. Hirshfield, Phys. Rev. ST Accel. Beams 9, 031301 (2006).

[29] G. V. Sotnikov, I. N. Onishchenko, J. L. Hirshfield, and T. C. Marshall, Probl. At. Sci. Technol. 49, 148 (2008) [http://vant.kipt.kharkov.ua/ARTICLE/VANT_2008_3/ article_2008_3_148.pdf].

[30] Kwang-Je Kim, Nucl. Instrum. Methods Phys. Res., Sect. A 275, 201 (1989).

[31] X. J. Wang, X. Qiu, and I. Ben-Zvi, Phys. Rev. E 54, R3121 (1996).

[32] X. J. Wang, M. Babzien, K. Batchelor, I. Ben-Zvi, R. Malone, I. Pogorelsky, X. Qui, J. Sheehan, J. Skaritka, and T. Srinivasan-Rao, Nucl. Instrum. Methods Phys. Res., Sect. A 375, 82 (1996). 